\title{
Recurrence Plot Analysis of Unsatured Polyester Samples Subjected to Contamination
}

\author{
Aysel Ersoy Yılmaz' ${ }^{1 D}$, Mehmet Murat İspirli² \\ 'Department of Electrical and Electronics Engineering, İstanbul University School of Engineering, İstanbul, Turkey \\ 2Department of Electrical and Electronics Engineering, Marmara University School of Technology, İstanbul, Turkey
}

Cite this article as: A. Ersoy Yılmaz, M. M. İspirli. "Recurrence Plot Analysis of Unsatured Polyester Samples Subjected to Contamination". Electrica, vol. 18, no. 1, pp. 13-18, 2018.

\begin{abstract}
Polymer materials that are used in outdoor enviroments are exposed to humidity and polution. In addition, some insulation materials installed in water or embeded to slush containing serious pollutants for a long time. These conditions adversely affect the dielectric properties of insulating materials. In this study, the prepared unsaturated polyester (UPE) samples are stored in an ionized water solution for 8, 24, and 168 hours. This ionized water solution is considered to represent the polluted water in the outdoor environments. Further, the dielectric behaviors of the prepared polyester samples are investigated using the inclined plane test (IPT) method, whereas the leakage current data that are recorded during the experiment are analyzed using the recurrence plot (RP) method. The results of applying the inclined plane test on the unsaturated polyester samples depict that the lifetime of the samples decreased with an increase in the period of storage in the ionized water solution. Further, the results of the recurrence plot analysis reveal that the recurrence plot point density also depicted a decrease with an increase in the period of storage in the ionized water solution.
\end{abstract}

Keywords: Inclined plane test, surface tracking, recurrence plot analysis, tracking failure

Address for Correspondence:

Mehmet Murat Ispirli

E-mail:

mispirli@marmara.edu.tr

Received: 18.06 .2017

Accepted: 30.12 .2017

C Copyright 2018 by Electrica

Available online at

http://dergipark.gov.tr/iujeee

DOI: $10.5152 /$ iujeee.2018.1803

\section{Introduction}

Today pollution is a key element which effects the quality of humans life and for electrical and electronics devices. Pollution can be gaseous, liquid or solid. Due to the increase of electrical device usage in every industrial sector the reliability and the continuity of the electric power systems gain importance. The intolerence to power outages force engineers to build more resistant materials against pollution and other enviromental factors. Pollution tests are become improtant and several test methods are introduced by several institutes such as IEC, VDE, ASTM, TSE, etc.

Polymers are widely used as insulating material in electrical systems due to their excellent dielectric properties [1-3]. Polymers are subjected to severe weather conditions such as rain, snow, storm, hail and environmental pollution under operating conditions [4]. Exposure of the material to a high electric field under these environmental conditions can cause the electrical insulation material to be degraded.

Progressive degradation of the surface of a solid insulating material by local discharges tend to form conducting or partially conducting paths [5]. Electrical tracking formed on the surface of polymeric materials is characterized by the formation of conductive paths [6]. The formation process of permanent conductive paths along the insulation surface under voltage stress is called tracking [7]. Due to pollution and humidity a conductive layer occurs on the surface of polymeric materials operating in the outdoor environment. When voltage is applied, leakage currents flow from this conductive layer. Heat is generated as a result of these flowing leakage currents. Along with the heat generated, a dry-band is formed on the insulation surface. The formation of the dry-band creates sparks which damage the insulation surface. Carbonization starts on the insulation surface due to these sparks. The resulting carbonization causes permanent discharges on the insulation surface. Due to permanent discharges, permanent 
conductive paths are formed on the insulation surface and these conductive paths spread with time. Finally, the insulation capability of surface between the electrodes completely is lost and breakdown initiated $[8,9]$. This phenomenon is known as electrical tracking failure.

Based on water absorption resistance polymeric materials can be classified into two class which are hydrophobicity or hydrophilicity. Hydrophobicity is one of the most useful properties of outdoor environment insulation materials [1]. If a material is hydrophobic, a conductive water layer does not form on the surface. Thus, surface discharges in the material are reduced. Energy loss is also reduced with reducing leakage currents flowing through the surface $[1,10]$. Unsaturated polyester (UPE) absorbs water in very small amounts to its structure. The amount of water absorbed to its structure are changed depends on the temperature and content of the water. In the study conducted by Dhakal et al., UPE samples were stored for 900 hours in water at room temperature and boiling water. As a result of this study, UPE samples absorbed about $0.5 \%$ water at room temperature and about $2 \%$ water at boiling water [11]. In a study conducted by Huang et al., samples are prepared using glass fiber fabric and unsaturated polyester absorbed about $1.5 \%$ of water after 21 days [12].

In this study, unsaturated polyester samples which are hydrophilic were stored in the electrolyte solution for 0, 8, 24, 168 hours respectively. These solution-waited samples were tested according to the IEC 587 Inclined Plane Test (IPT) method. After the tests, weight loss in the samples were measured. The recorded leakage current data was evaluated by the recurrence plot (RP) method, which is frequently used in the analysis of nonlinear time series. Using these results, the change of the dielectric properties of the polyester samples with storage time in the electrolyte solution were examined.

\section{Material and Method}

There are different test standards issued by IEC (International Electrotechnical Commission) and ASTM (American Society for Testing and Materials) organizations to evaluate electrical tracking resistance of polymer samples. In this study, Inclined Plane Test method determined by these organizations was used. IEC 60587 and ASTM D2303 are test method standards set by IEC and ASTM organizations, respectively. This test method was first published in 1964 as ASTM D2303 [13]. Then test method was later accepted by IEC and published in 1977 as IEC 60587 [14]. The test standard was last revised by IEC and ASTM organizations in 2007 and 2013, respectively [5,16]. Despite the small differences between these two test standards, they are based on the same theory. A schematic representation of the experimental setup based on these standards is shown in Figure 1. $4 \mathrm{kV}$ AC voltage was applied to the samples. A resistance of $33 \mathrm{k} \Omega$ was connected in series to the test sample. The flow rate of the electrolytic liquid from the surface was set at $36 \mathrm{ml} / \mathrm{h}$.

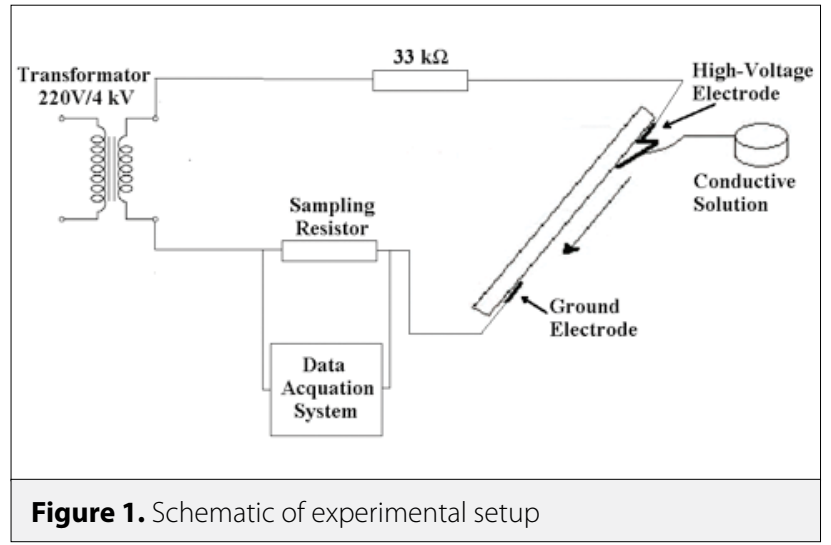

According to the IEC and ASTM test standarts by the following conditions satisfied in the inclined plane test, tracking failure is occured $[5,16]$;

- The value of the leakage current flowing from the sample surface is over $60 \mathrm{~mA}$ for $2 \mathrm{~s}$.

- The track length on the sample surface is $25 \mathrm{~mm}$ or more from the ground electrode

- $\quad$ Erosion on the sample surface $[5,16]$.

All the samples used in this study were prepared by using $0.25 \%$ methyl-ethyl-ketone-peroxide (MEKP) and $0.25 \%$ accelerator (cobalt) by weight in polyester resin under the same laboratory conditions. Test samples were molded in dimensions of $120 \times 50 \times 9 \mathrm{~mm}$.

\section{Recurrence Plot Analysis}

The recurrence plot method is an advanced nonlinear data analysis method. This method was presented in 1981 by Maizel and Lenk as a method of visual model analysis of a series of genetic nucleotides $[17,18]$. Fundamental structures of the recurrence plot analysis were developed by Eckman et al. [19]. Recurrence plot is a representation of the recurred behavior of the values of the $\mathrm{m}$-dimensional phase space in the two-dimensional phase space. Both axis on the recurrence plots are time. Recurrence plots are a practical way of processing data since it is a graphical framework for extracting information from the time series. $[17,20]$. Leakage currents are chaotic signals. For this reason, the application of the recurrence plot technique is very useful for analyzing the leakage currents $[17,21]$.

Dynamic systems with non-linear characteristics can be sampled using method in $[17,22]$. Recurrence plot method has the advantage of giving accurate results even for very short and unstable data $[17,23]$. According to Takens' embedding theorem, the time series is appropriately reconstructed as follows $[17,24]$.

$X_{(i)}=[x(i), x(i+\tau), \ldots, x(i+(m-1) \tau)], i=1,2,3 .$. 
Where;

$\mathrm{X}_{(\mathrm{i})}$ : Embedding Vector,

$m$ : Embedding Dimension,

$\tau$ : Delay Time,

$\mathrm{x}(\mathrm{i})$ : Time series.

In this study, $x(i)$ time series are defined as the leakage current data recorded from the system. Defining a function obtained from a time series in a dynamic characteristic as recurrence plot series is as follows.

$R_{i, j}=H\left(\varepsilon-\left\|X_{(i)}-X_{(j)}\right\|_{2}\right), X_{(i)}, X_{(j)} \in R^{m}, i, j \in(1, M)$

Where;

$\mathcal{E}$ :Threshold value,

\|.. : Euclidean, maximum or manhattan norm,

$H(x)$ : Heaviside function

$M$ : Number of $X_{(i)}$

Threshold value selection is very important when analyzing with the recurrence plot technique. The standard deviation of the time series is used when the threshold value $(\varepsilon)$ is determined. If the threshold value is chosen extreme, irrelevant points will appear on the plot. If the threshold value is chosen too small, it will cause to loss of some data that might give the right result $[17,25]$. Selected threshold value and the values of the data in the embedding vector are processed in the Heaviside unit step function. As a result of this operation, the elements with value 1 are drawn with black in the recurrence plot, while those in value 0 are drawn with white.

\section{Results}

Prepared UPE samples in laboratory conditions were aged by waited in solution containing $0.1 \% \mathrm{NH}_{4} \mathrm{Cl}$ and $0.2 \%$ Triton $\mathrm{X}-100$. While this aging was conducted, 3 groups of samples were waited in this solution for 8,24 and 168 hours, respectively. Additionally alternative sample group under the same conditions was prepared without waiting session. 3 sample groups are exposed to aging process and a sample group was not exposed to aging process which were evaluated for surface tracking performance with the proposed experimental setup.

\section{Surface Tracking Results}

The samples aged in the solution were removed from the solution and dried. After drying, weights of the samples were measured with an analytical balance having a sensitivity of 0.1 mg. With this weights, the samples were compared with their weight prior to being stored in solution. Weight gains in the samples are given as a percentage in Figure-2. By the computational approach, the average of each sample group is taken within their scopes. UPE samples absorbed water $0.011 \%$, $0.017 \%$ and $0.089 \%$ of their mass in solution at 8,24 and 168 hours respectively. The water absorption rate of the UPE is decreased with the increased waiting time in the solution. Due to the saturation rate of the sample increases with time.

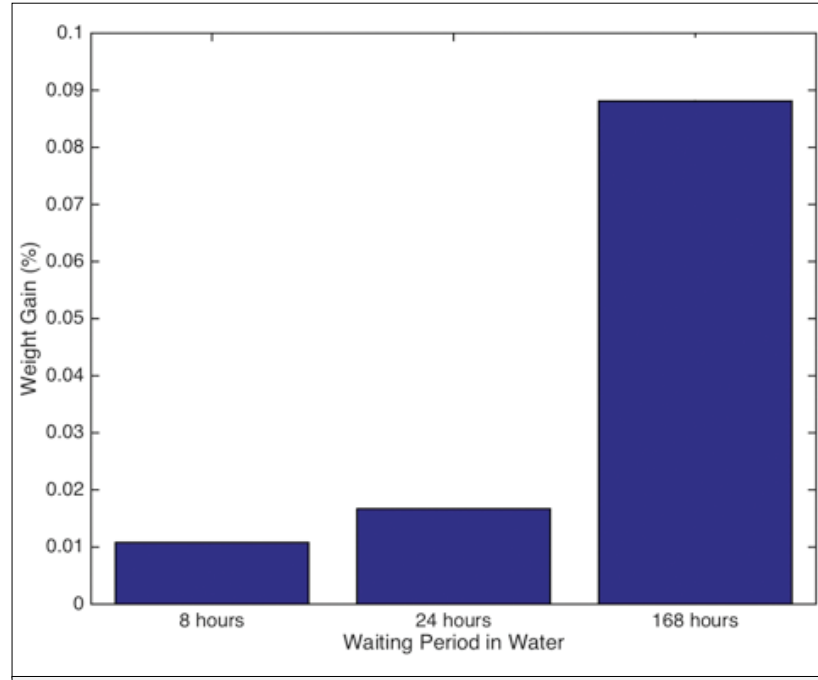

Figure 2. Weight gain of samples according to waiting time in solution

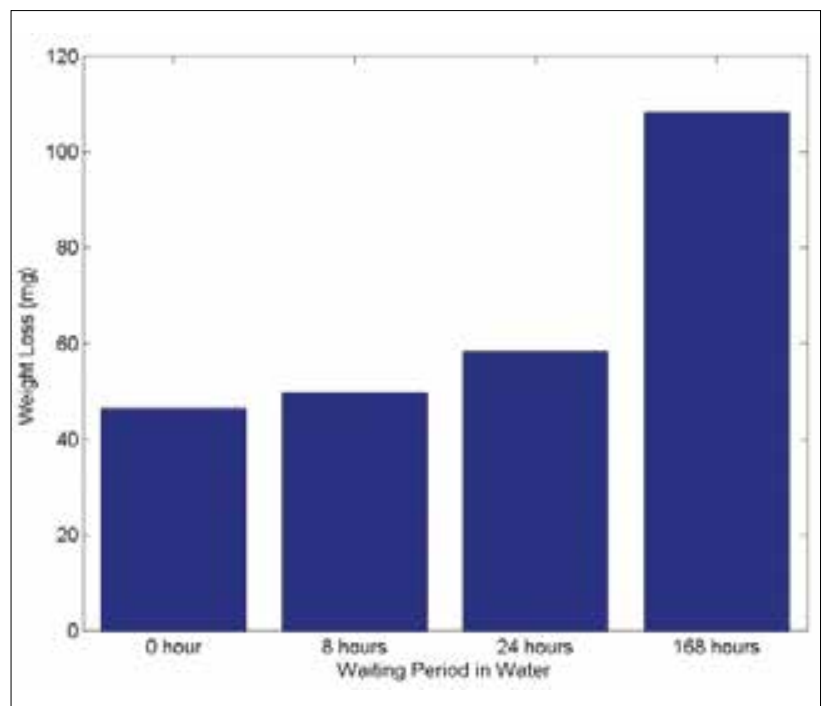

Figure 3. Weight loss of samples according to waiting time in solution

Before the test, sample weight was taken with a digital scale. Then the samples were tested on the prepared experimental setup according to IEC 587 standard. After each test, residues of carbon tracks formed on the sample surface were cleaned. Finally the sample weight was measured again. Averages of weight loss within each sample group were taken. The weight losses occurring in the sample groups according to the waiting times in the solution are given in Figure 3. Weight loss occurred in the samples waited for 8 hours in solution was about $7.5 \%$ higher than samples that were not waited. Weight loss of the sample that was waited for 24 hours in solution was about $25 \%$ higher than the sample that was not waited. Weight loss of the sample waited in the solution for 1 week (168 hours) is 2.3 times the weight loss of the sample that was not waited. 


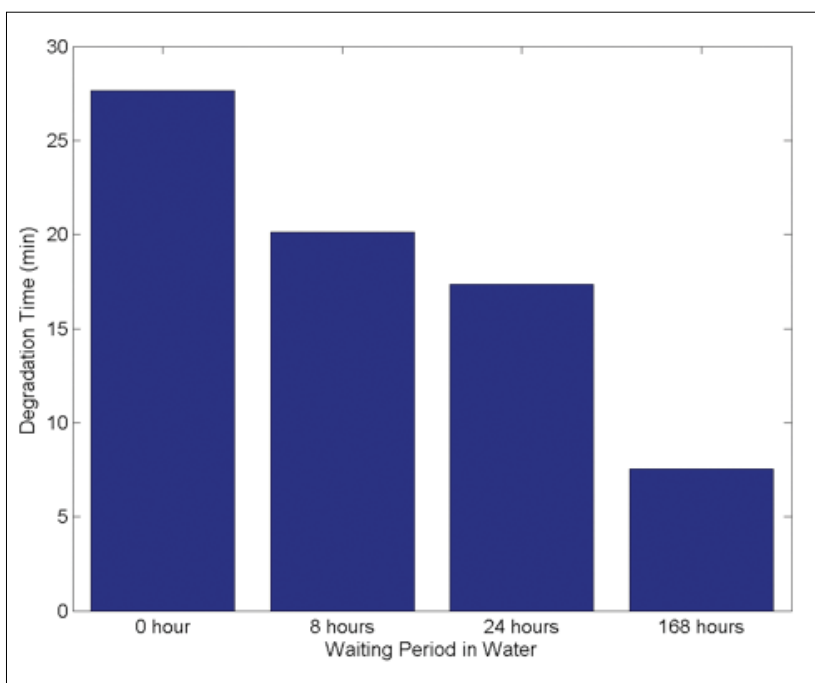

Figure 4. Degradation Time of samples according to waiting time in solution

In each test group the degradation time of samples were noted. When this procedure was carried out, the average of the degradation times of each test group is noted along with weight loss. The degradation time of the sample groups according to the waiting times in the solution are given in Figure 4. According to test results, the degradation time of the sample group that was waited for 8 hours in solution decreased by about 27\% compared to the sample group that was not waited. The degradation time of sample groups waited in solution for 24 hours and 1 week (168 hours) decreased by $37 \%$ and $72.5 \%$, respectively, when compared to the sample group that was not waited.

The tracking formed on the surface of the samples as a result of the experiments based on the ASTM D2303 standard are shown in Figure 5.

\section{Recurrence Plot Results}

For the recurrence plot analyze the leakage current flowing from the sample surface was recorded by Analog/Digital conversion device. The recorded data was analyze using the recurrence plot method. The relationship of leakage current data and waiting time of samples in solution was analyzed by this method. The last 3 seconds of the test procedure before the degradation of the sample was investigated by this analysis. The power frequency components have been filtered when the recurrence plot analysis is done. Fourier transform is used for eliminated $50 \mathrm{~Hz}$ Components $(48-52 \mathrm{~Hz})$. In this analysis, embedding dimensiın $(m)$ is 3 , delay time $(\tau)$ is 8 and threshold is $0.3 \cdot \sigma . \sigma$ is standart deviation of time series. Figure 6 shows the recurrence plots of samples from all sample groups. When the RP of the sample unwaited in the solution given in Figure 6a is compared with the RP of the sample waited in the solution for 8 hours in Figure $6 b$, it is seen that the black point density is more intense in the sample that was not waited. In other words, the density of the white space is higher in the RP belonging to

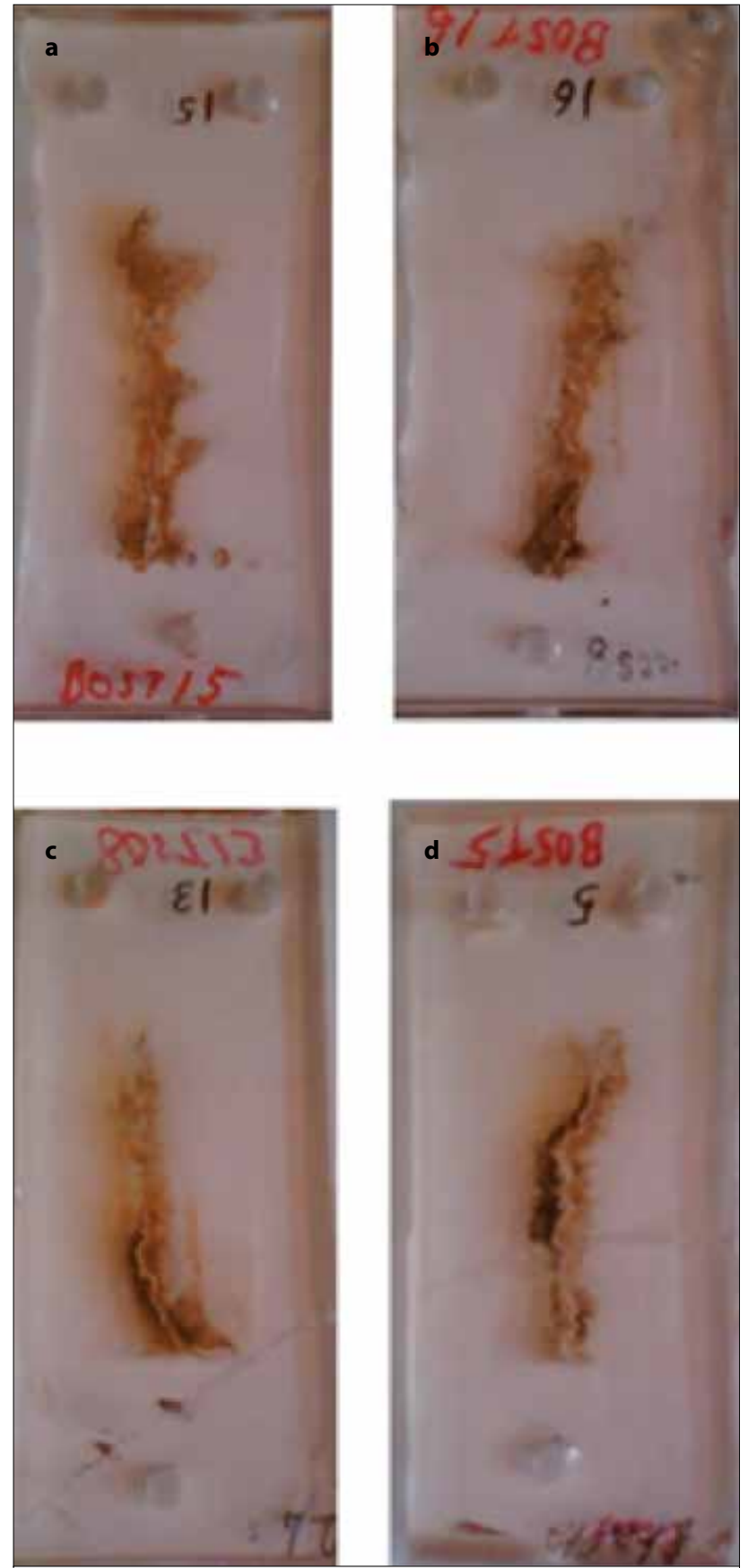

Figure 5. a-d. Observed tracking on UPE samples a) unwaited b) waited for 8 hours c) waited for 24 hours d) waited for 168 hours

the sample waited in the solution for 8 hours. Likewise, when the RP of the sample waited in the solution for 8 hours in Figure $6 \mathrm{~b}$ is compared to the RP of the sample waited in the solution for 24 hours in Figure $6 c$, the black point density in the RP of the sample waited in the solution for 24 hours is less. When the RPs of all the samples were compared that were unwaited in the solution and waited in the solution for 8, 24, and 168 hours, the black point density was the maximum on the sample that was unwaited and it was the minimum on the sample that was 

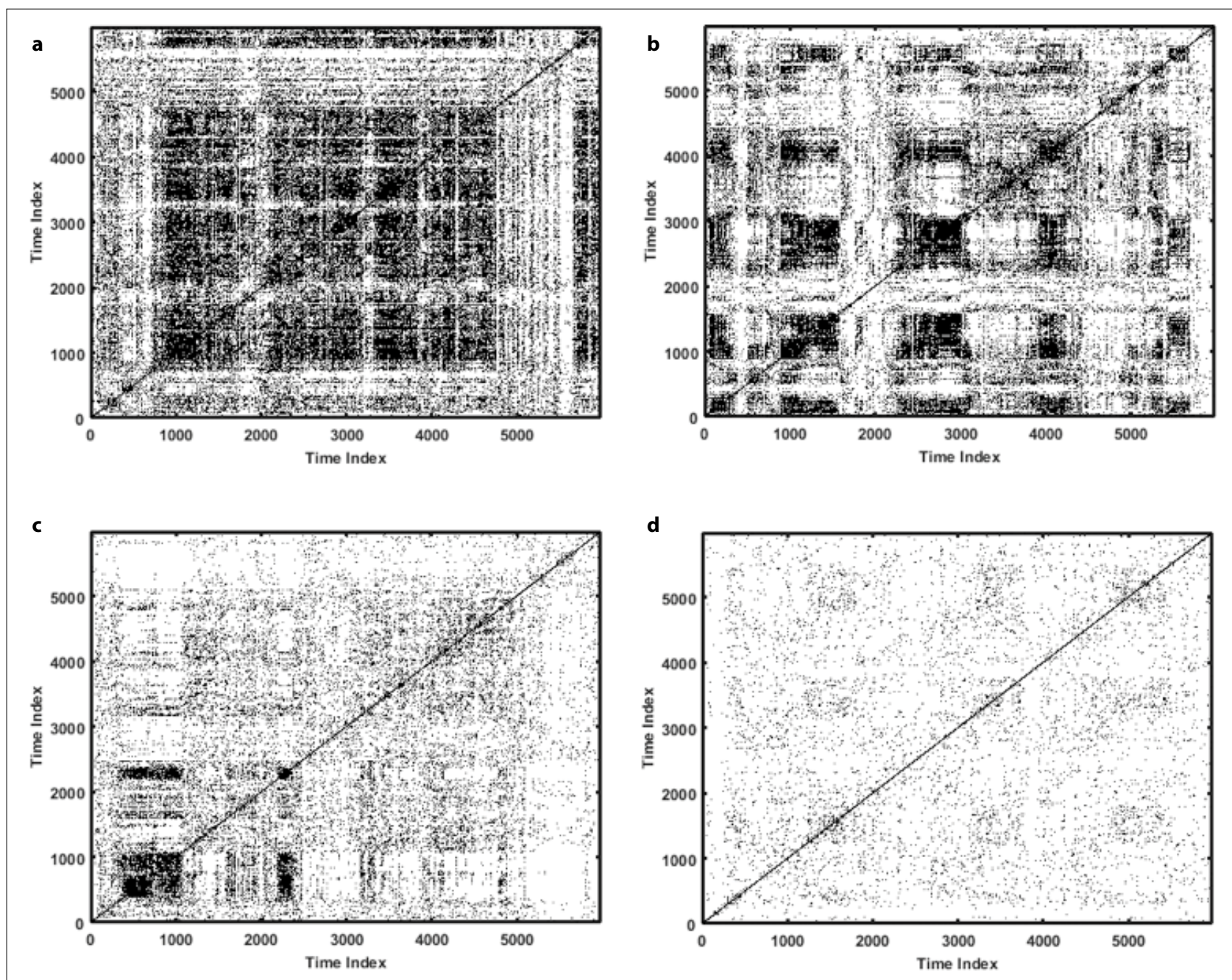

Figure 6. a-d. Recurrence Plots (a) unwaited (b) waited for 8 hours (c) waited for 24 hours (d) waited for 168 hour

waited in the solution for 168 hours. Namely, the density of the white space is the highest in the RP of the sample, which was waited in solution for 168 hours and shown in Figure $6 \mathrm{~d}$.

\section{Conclusions}

In this study, unsatureted polyester samples prepared under laboratory conditions were tested according to Inclined Plane Test method. As a result of the tests, weight loss in the samples were measured. The recorded leakage current data from the sample surface during the tests was analyzed by the recurrence plot method. As a result of these information;

1. Weight loss in the samples increased with increasing the waiting time of the samples in the solution

2. The degradation times of the samples were shortened with increasing the waiting time of the samples in the solution,

3. By increasing the waiting time of the samples in the solution, RP Point Density was decreased, White Space was increased. Thus, by increasing the waiting time of the samples in the water, the amplitude of the leakage currents flowing through the sample surface was increased.

4. The increase in the waiting time of the samples in the solution adversely affected the dielectric performance of the samples.

When all these results are evaluated, resistance to tracking of UPE is inversely proportional with waiting time in solution. Precautions like coating to prevent water absorption must be taken when unsaturated polyester materials used under wet and dirty conditions.

\section{References}

1. J. P Reynders, I. R. Jandrell, S. M. Reynders, "Review of aging and recovery of silicone rubber insulation for outdoor use," IEEE Transactions on Dielectrics and Electrical Insulation, vo. 6, no. 5, pp. 620-631, 1999. [CrossRef]

2. R. S. Gorur , E. A. Cherney, R. Hackam, T. Orbeck, "The electrical performance of polymeric insulating materials under accelerated 
aging in a fog chamber," IEEE Transactions on Power Delivery, vol. 3, no. 3, pp. 1157-1164, 1988. [CrossRef]

3. R. G. Houlgate, D. A. Swift, A. Cimador, F. Purbaix, G. Marrone, P. Nicolini, "Field Experience and Laboratory Research on Composite Insulators for Overhead Lines," CIGRE 1986.

4. W. T. Shugg, and K.N. Mathes, "Handbook of electrical and electronic insulating materials", Vol. 995, IEEE press, New York, 1995.

5. Electrical insulating materials used under severe ambient conditions - Test methods for evaluating resistance to tracking and erosion, IEC 60587:2007 International Standard, 3rd ed., 2007.

6. R. F. Da Silva and S. V. Filho, "Analysis of electrical tracking by energy absorption during surface discharge in polymeric materials," IEEE Transactions on Dielectrics and Electrical Insulation, vol. 23, no. 1, pp. 501-506, 2016. [CrossRef]

7. K. Y. Lau and M.A.M Piah, "Polymer nanocomposites in high voltage electrical insulation perspective: a review," Malaysian Polymer Journal, vol. 6, no. 1, pp. 58-69, 2011.

8. L. Zhang, “Electrical Tracking Over Solid Insulating Materials for Aerospace Applications,"Ph.D. Thesis, Diss. University of Manchester, 2011.

9. A. Mazen, A. Hussein, E. Ahdab, R. Roshdy, "High-Voltage Engineering: Theory and Practice, Revised and Expanded". Marcel Dekker Inc, New York, 2000.

10. H. Janssen, "The loss and recovery of hydrophobicity on silicone rubber surfaces." 10th International Symposium on High Voltage Engineering, Montreal, Canada. 1997.

11. H. N. Dhakal, Z.Y. Zhang, M.O.W. Richardson, "Effect of water absorption on the mechanical properties of hemp fibre reinforced unsaturated polyester composites," Composites Science and Technology, vol. 67, no. 7, pp. 1674-1683, 2007. [CrossRef]

12. G. Huang, H. SUN, 1Effect of water absorption on the mechanical properties of glass/polyester composites", Materials \& design, vol. 28, no. 5, pp. 1647-1650, 2007. [CrossRef]

13. G. R. Mitchell, "Present Status of ASTM Tracking Test Methods," Journal of Testing and Evaluation vol. 2, no.1, pp. 23-31, 1974. [CrossRef]

14. Test method for evaluating resistance to tracking and erosion of electrical insulating materials used under severe ambient conditions, IEC 60587:1977 International Standard, 1st ed., 1977.
15. M. S. Naidu, V Kamraju, "High voltage engineering," Tata McGraw-Hill Education, New Delhi, India, 2013.

16. ASTM D2303-13, Standard Test Methods for Liquid-Contaminant, Inclined-Plane Tracking and Erosion of Insulating Materials, ASTM International, West Conshohocken, PA, 2013, www.astm.org.

17. M.M. İspirli and A. Ersoy Yılmaz, "Tracking failure and recurrence plot analysis for three different levels in the discharge on epoxy resin," Electrical, Electronics and Biomedical Engineering (ELECO), National Conference on. IEEE. pp. 349-353, 2016.

18. J.V. Maizel and P.L. Robert, "Enhanced graphic matrix analysis of nucleic acid and protein sequences," Proceedings of the National Academy of Sciences, vol. 78, no. 12, pp. 7665-7669, 1981. [CrossRef]

19. J-P. Eckmann, S. O. Kamphorst, D. Ruelle. "Recurrence plots of dynamical systems," EPL (Europhysics Letters), vol. 4 no. 9, pp. 973977, 1987. [CrossRef]

20. Y. Liu and B. X. Du. "Recurrent plot analysis of leakage current on flashover performance of rime-iced composite insulator," IEEE Transactions on Dielectrics and Electrical Insulation, vol. 17, no.2, pp. 465-472, 2010. [CrossRef]

21. A. Wolf, J.B. Swift, H.L. Swinney, J.A. Vastanox, "Determining Lyapunov exponents from a time series," Physica D: Nonlinear Phenomena, vol. 16, no. 3, pp. 285-317, 1985. [CrossRef]

22. B. X. Du, Y. Gao, Y. Liu. "Effects of Gamma-Ray Irradiation on Tracking Failure of Polymer Insulating Materials." Nuclear Power-operation, safety and enviroment, Dr. Pavel Tsvetkov (Ed.), In Tech, 2011, DOI: 10.5772/17263 [CrossRef]

23. N. Marwan, M.C. Romano, M. Thiel, J. Kurths, "Recurrence plots for the analysis of complex systems," Physics Reports, vol. 438, no. 5, pp. 237-329, 2007. [CrossRef]

24. F. Takens, "Detecting strange attractors in turbulence", In Dynamical systems and turbulence, Warwick 1980 (pp. 366-381). Springer Berlin Heidelberg, 1981. [CrossRef]

25. B. X. Du, Y.G. Guo, Y. Liu, L Tian, "Effects of adding nanofiller on DC tracking failure of epoxy/MgO nano-composites under contaminated conditions," IEEE Transactions on Dielectrics and Electrical Insulation, vol. 21, no. 5, pp. 2146-2155, 2014. [CrossRef]

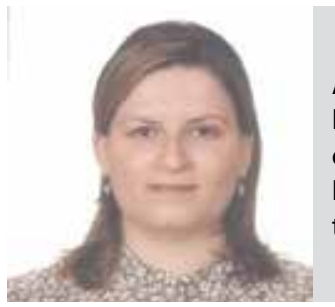

Aysel Ersoy Yılmaz was born in Eskişehir. She received her M.Sc. and Ph.D. degrees in Electrical and Electronics Engineering from Istanbul University in 2003 and 2007, respectively. She studied as a post doctorate researcher at Kettering University in USA between 2008 and 2009. Currently she is working as Assistant Professor in Electrical - Electronics Engineering Department of İstanbul University. Her research interests are high voltage techniques, dielectric materials and renewable energy applications.

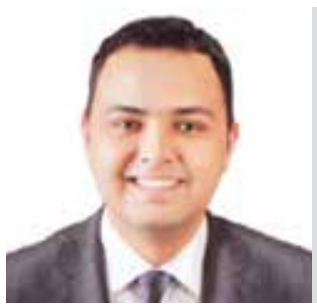

Mehmet Murat İspirli was born in Çorum on May 14, 1991. He received his B.Sc. degrees in Electrical and Electronics Engineering from İstanbul University in 2015. He is an M.Sc. student at İstanbul University at Electrical-Electronics Engineering Department. Currently, he is working as Research Assistant in Electrical - Electronics Engineering Department of Marmara University. His research interests are high voltage technique, high voltage insulation materials, discharge phenomena, numerical analysis of electrostatic fields, protection against overvoltages and renewable energy materials. 